\title{
Symmetric Shannon capacity is the independence number minus 1
}

\author{
Tamás Terpai ${ }^{1}$ (D)
}

Accepted: 1 March 2021 / Published online: 10 December 2021

(c) The Author(s) 2021

\section{Abstract}

A symmetric variant of the Shannon capacity of graphs is defined and computed.

Keywords Shannon capacity · Independence number · Graph product

Mathematics Subject Classification 05C69 · 05C76

\section{Introduction and motivation}

The Shannon capacity $\Theta(G)$ (introduced in 1956 by Shannon [5]) is a notoriously hard-tocompute graph parameter defined as the asymptotic growth rate of the independence number of strong direct powers of a graph $G$ :

$$
\Theta(G)=\lim _{k \rightarrow \infty}\left(\alpha\left(G^{\bigotimes k}\right)\right)^{1 / k}
$$

(the limit always exists due to the supermultiplicativity of $\alpha\left(G^{\bigotimes k}\right)$ ). Even for the 5-cycle the exact value was only determined in 1979 by Lovász [4], and the exact value for the 7 -cycle is unknown (asymptotic results exist for large odd cycles, see Bohman [2,3]). As to approximation, Alon and Lubetzky [1] show that the naïve strategy of estimating $\Theta(G)$ by $\alpha\left(G^{\bigotimes k}\right)$ for any fixed $k$ can produce unboundedly high relative error; in fact, there may be arbitrarily high polynomial jumps in the value of $\alpha\left(G^{\bigotimes k}\right)$ at any finite set of $k$ 's.

In this paper, we use a modification of Shannon capacity where instead of the strong direct power $G^{\bigotimes k}$ we consider its quotient $G[k]=G^{\bigotimes k} / S_{k}$ by the natural index-permuting action of the symmetric group $S_{k}$. Since the size of $G[k]$ (and consequently of $\alpha(G[k])$ as well) grows only polynomially, we define

$$
F(G)=\lim _{k \rightarrow \infty} \frac{\log \alpha(G[k])}{\log k}
$$

as a measure of asymptotic growth of $\alpha(G[k])$. Our main result is the following:

Tamás Terpai

terpai@math.elte.hu

1 Department of Analysis, ELTE Eötvös Loránd University, Pázmány Péter sétány 1/C, Budapest 1117, Hungary 
Theorem For any finite graph $G$ we have $F(G)=\alpha(G)-1$.

Section 2 fixes our notation and Section 4 contains the proof of the Theorem. Section 3 contains a treatment of the particular case of the 5-cycle in a way that does not seem to generalize to all graphs but yields more precise intermediate estimates.

\section{Setup}

Let $G$ be a simple graph on the vertex set $V=\left\{v_{1}, \ldots, v_{n}\right\}$. Then the vertex set $V[k]$ of the graph $G[k]=G^{\bigotimes k} / S_{k}$ consists of partitions of $k$ into $n$ labeled nonnegative integers:

$$
V[k]=\left\{f: V \rightarrow \mathbb{Z}_{\geq 0}: f\left(v_{1}\right)+\cdots+f\left(v_{n}\right)=k\right\} .
$$

We will consider the elements of $V[k]$ also as equivalence classes of maps from a set of cardinality $k$ to $V$ with two maps being equivalent if they only differ in a permutation of the $k$ elements-configurations of $k$ identical pebbles in the vertices of $G$. Two vertices $f, g \in V[k]$ are connected if one can move some pebbles in the configuration corresponding to $f$ to neighbours in $G$ to obtain the configuration corresponding to $g$ (were the pebbles distinguishable, this construction would give the strong direct product $G^{\bigotimes k}$ ).

A priori it is not clear whether the limit defining $F(G)$ exists at all: in contrast to the case of distinguishable pebbles, there is no obvious superadditivity property for $\log \alpha(G[k])$ that would ensure convergence. It is however easy to see that

$$
\alpha(G[k]) \geq\left(\begin{array}{c}
k+\alpha(G)-1 \\
\alpha(G)-1
\end{array}\right)
$$

- the configurations supported on a fixed independent set $W \subset V$ are clearly independent in $G[k]$-and that

$$
\alpha(G[k]) \leq\left(\begin{array}{c}
k+\theta(G)-1 \\
\theta(G)-1
\end{array}\right)
$$

where $\theta(G)$, the clique covering number of $G$, is the minimal number of cliques covering the vertices of $G$-for any clique covering $V=V_{1} \cup \cdots \cup V_{\theta}$ whenever the configurations $f$, $g \in V[k]$ have the same number of pebbles on the vertices of all $V_{j}$, they can be moved into one another by using only the edges within the $V_{j}$. This argument shows that if $\theta(G)=\alpha(G)$, then $\alpha(G[k])=\left(\begin{array}{c}k+\alpha(G)-1 \\ \alpha(G)-1\end{array}\right)$ and $F(G)=\alpha(G)-1$ does indeed hold.

The simplest graph for which $\theta(G)>\alpha(G)$ (and thus the estimates above do not determine $\alpha(G[k]))$ is the 5-cycle $C_{5}$; the following section discusses this particular graph.

\section{The case of $C_{5}$}

It can be checked that for $k \leq 9$ we have $\alpha\left(C_{5}[k]\right)=k+1$, but for general $k$ we can only prove the following, weaker result:

Proposition 3.1 For any natural number $k$ we have $\alpha\left(C_{5}[k]\right) \leq\left\lfloor\frac{5(k+2)(k+1)}{2(k+5)}\right\rfloor$.

Proof For simplicity let us index the vertices of $C_{5}$ by natural numbers from 1 to 5 in such a way that vertices $v_{j}$ and $v_{j+1}$ are connected by an edge labelled $e_{j}$ for $j=1, \ldots, 4$, and the vertices $v_{1}$ and $v_{5}$ are also connected by the edge $e_{5}$. 
Fig. 1 Labelling of $C_{5}$

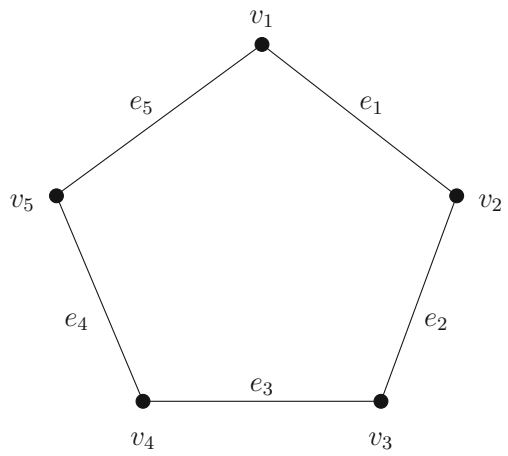

Consider edge configurations of weight $k$ on $C_{5}$ : functions $\psi: E\left(C_{5}\right) \rightarrow \mathbb{Z}_{\geq 0}$ such that $\psi\left(e_{1}\right)+\cdots+\psi\left(e_{5}\right)=k$, also considered as ways to distribute $k$ indistinguishable pebbles among the edges of $C_{5}$. We declare an edge configuration $\psi$ and a vertex configuration $f$ to be adjacent if one can obtain $\psi$ from $f$ by moving each pebble to an adjacent edge; this defines a (bipartite) graph structure on the set of vertex and edge configurations. Note that if $f$ and $g$ are vertex configurations, then they are neighbours in $C_{5}[k]$ exactly if there exists an edge configuration $\psi$ adjacent to both of them. As a consequence, if $A \subset V[k]$ is an independent set, then the sets of edge configurations adjacent to the elements of $A$ are disjoint.

From this fact we get a bound on $|A|$ : if every vertex configuration is adjacent to at least $m$ edge configurations, and their total size cannot exceed $\left(\begin{array}{c}k+4 \\ 4\end{array}\right)$, the total number of edge configurations of weight $k$, then $|A| \leq \frac{\left(\begin{array}{c}k+4 \\ 4\end{array}\right)}{m}$. Unfortunately, there are vertex configurations that are adjacent to few edge configurations: for example, only $k+1$ edge configurations are adjacent to the vertex configuration where all the pebbles are assigned to the same vertex, and this makes the obtained upper bound on $|A|$ too high. To circumvent this problem, we count edge configurations with weight. Firstly, we count only edge configurations that assign zero weight to at least one pair of non-adjacent edges. Let $S=S_{1} \cup S_{2} \cup S_{3} \cup S_{4} \cup S_{5}$ denote the set of such edge configurations, where $S_{j}$ is the set of those edge configurations that assign zero weight to edges $e_{j-1}$ and $e_{j+1}$ (with indices considered modulo 5). Finally, as the sets $S_{j}$ are not disjoint, we shall consider each edge configuration weighted by the number of sets $S_{j}$ that contain it.

Now let $f \in V[k]$ be an arbitrary vertex configuration. Edge configurations in $S_{1}$ that are adjacent to $f$ have no pebbles on edges $e_{2}$ and $e_{5}$ and hence must be formed by moving all pebbles in $v_{1}$ and $v_{2}$ to the edge $e_{1}$, all pebbles in $v_{3}$ to the edge $e_{3}$ and all pebbles in $v_{5}$ to the edge $e_{4}$. The only freedom left is the distribution of the pebbles in $v_{4}$ to $e_{3}$ and $e_{4}$; the number of such distributions is $f\left(v_{4}\right)+1$. Repeating this argument for $S_{2}$ to $S_{5}$, we count $f\left(v_{1}\right)+1+\cdots+f\left(v_{5}\right)+1=k+5$ edge configurations adjacent to $f$, each as many times as there are sets $S_{j}$ that contain it. The total weight of the edge configurations in $S$ is $\left|S_{1}\right|+\cdots+\left|S_{5}\right|=5\left(\begin{array}{c}k+2 \\ 2\end{array}\right)$, hence there cannot be more than $\left\lfloor\frac{5\left(\begin{array}{c}k+2 \\ 2\end{array}\right)}{k+5}\right\rfloor=\left\lfloor\frac{5(k+2)(k+1)}{2(k+5)}\right\rfloor$ pairwise non-neighbouring vertex configurations, as claimed.

As an immediate corollary, we get

Corollary $3.2 F\left(C_{5}\right)=1$.

Open question 3.1 Does $\alpha\left(C_{5}[k]\right)=k+1$ hold for all $k$ ? 


\section{General case}

The main Theorem is the immediate consequence of the trivial lower bound and the following estimate:

\section{Proposition 4.1 For any finite graph $G$ we have $\alpha(G[k])=O\left(k^{\alpha(G)-1}\right)$.}

Proof We prove our statement by induction on the cardinality of $V$. For $|V|=1$ the claim is trivial. Assume now that the statement holds for all graphs with less vertices than $|V|$, and let $H \subset V[k]$ be an arbitrary independent vertex set in $G[k]$. Partition $H$ into pieces $H_{1}$, $\ldots, H_{n}$ such that in $H_{j}$ the vertex $v_{j}$ carries the greatest weight; in particular, this weight is at least $\frac{k}{n}$.

Denote by $N\left(v_{j}\right) \subset V$ the union of the vertex $v_{j}$ and its neighbours in $G$. Each $H_{j}$

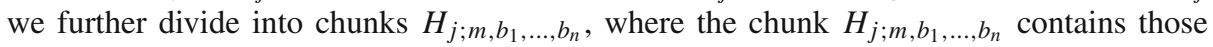
elements of $H_{j}$ in which the total weight of $v_{j}$ and its neighbours is $m$ and for all $i \leq n$ the weight of the vertex $v_{i}$ falls into the interval $\left[b_{i} \frac{k}{n^{2}},\left(b_{i}+1\right) \frac{k}{n^{2}}\right)$. The index $j$ can take $n$ different values, $m$ can take $k+1$ different values, and each $b_{i}$ can be assumed to be between 0 and $n^{2}$ (the rest are always empty), so altogether we get at most $n(k+1)\left(n^{2}+1\right)^{n}=O(k)$ chunks (with an implied dependence on $n$ ). Pick now an arbitrary chunk $H_{j ; m, b_{1}, \ldots, b_{n}}$ and consider its elements as configurations on $G \backslash N\left(v_{j}\right)$ (with the weights on the elements of $N\left(v_{j}\right)$ omitted). These configurations all have weight $k-m$ and we claim that they form an independent set in $\left(G \backslash N\left(v_{j}\right)\right)[k-m]$. Indeed, if two configurations $f$ and $g$ in $H_{j ; m, b_{1}, \ldots, b_{n}}$ either coincide or are neighbours in $\left(G \backslash N\left(v_{j}\right)\right)[k-m]$, then there exists a pebble transport from $f$ to $g$ on $G \backslash N\left(v_{j}\right)$. On the other hand, there exists a pebble transport from $f$ to $g$ on $N\left(v_{j}\right)$ as well: for each vertex $v_{i}$ that is a neighbour of $v_{j}$ we send $f\left(v_{i}\right)-g\left(v_{i}\right)$ pebbles from $v_{i}$ to $v_{j}$ if $f\left(v_{i}\right)>g\left(v_{i}\right)$, and we send $g\left(v_{i}\right)-f\left(v_{i}\right)$ pebbles from $v_{j}$ to $v_{i}$ if $g\left(v_{i}\right)>f\left(v_{i}\right)$. Combining these two pebble transports yields a pebble transport from $f$ to $g$.

This means that the chunk $H_{j ; m, b_{1}, \ldots, b_{n}}$ has size at most $O\left(k^{\alpha\left(G \backslash N\left(v_{j}\right)\right)-1}\right)$ by the induction hypothesis. But any independent set in $G \backslash N\left(v_{j}\right)$ can be extended by $v_{j}$ to form an independent set in $G$, hence $\alpha\left(G \backslash N\left(v_{j}\right)\right) \leq \alpha(G)-1$. Summing this estimate for all the $O(k)$ chunks we get that $|H|=O\left(k^{\alpha(G)-1}\right)$, proving the induction step.

Open question 4.1 Does $\alpha(G[k])=\left(\begin{array}{c}k+\alpha(G)-1 \\ \alpha(G)-1\end{array}\right)$ or at least

$$
\lim _{k \rightarrow \infty} \frac{\alpha(G[k])}{k^{\alpha(G)-1}}=\frac{1}{(\alpha(G)-1) !}
$$

hold?

Acknowledgements The author is grateful to Péter Frenkel for coming up with the definition of symmetric Shannon capacity and to Endre Csóka for organizing the Siófok Workshop on Graphs and Graph Limits where the related questions were disseminated.

Funding Open access funding provided by Eötvös Loránd University.

Open Access This article is licensed under a Creative Commons Attribution 4.0 International License, which permits use, sharing, adaptation, distribution and reproduction in any medium or format, as long as you give appropriate credit to the original author(s) and the source, provide a link to the Creative Commons licence, and indicate if changes were made. The images or other third party material in this article are included in the article's Creative Commons licence, unless indicated otherwise in a credit line to the material. If material is not included in the article's Creative Commons licence and your intended use is not permitted by statutory regulation or exceeds the permitted use, you will need to obtain permission directly from the copyright holder. To view a copy of this licence, visit http://creativecommons.org/licenses/by/4.0/. 


\section{References}

1. N. Alon, E. Lubetzky, The Shannon capacity of a graph and the independence numbers of its powers. IEEE Trans. Inf. Theory 52, 2172-2176 (2006)

2. T. Bohman, A limit theorem for the Shannon capacities of odd cycles I. Proc. Am. Math. Soc. 131, 35593569 (2003)

3. T. Bohman, A limit theorem for the Shannon capacities of odd cycles II. Proc. Am. Math. Soc. 133, 537-543 (2004)

4. L. Lovász, On the Shannon capacity of a graph. IEEE Trans. Inf. Theory 25(1), 1-7 (1979)

5. C.E. Shannon, The zero-error capacity of a noisy channel. IRE Trans. Inf. Theory 2(3), 8-19 (1956)

Publisher's Note Springer Nature remains neutral with regard to jurisdictional claims in published maps and institutional affiliations. 